\title{
La « Section Mauzin »
}

\section{René Terrasse}

\section{OpenEdition}

\section{Journals}

Édition électronique

URL : https://journals.openedition.org/rhcf/1881

DOI : 10.4000/rhcf.1881

Éditeur

Rails \& histoire

Édition imprimée

Date de publication : 1 juin 2003

Pagination : 96-105

ISBN : 0996-9403

ISSN : 0996-9403

Référence électronique

René Terrasse, "La «Section Mauzin » », Revue d'histoire des chemins de fer [En ligne], 27 | 2003, mis en ligne le 14 janvier 2015, consulté le 22 avril 2022. URL : http://journals.openedition.org/rhcf/1881 DOI : https://doi.org/10.4000/rhcf.1881 


\section{René TERRASSE}

\section{La "Section Mauzin"}

Ainsi l'a-t-on longtemps appelée, du nom de son créateur, alors que son nom officiel était «Section des essais et recherches ». J'y entrai en pleine Occupation, le 20 octobre $1943^{1}$ et y étais encore ( $j$ 'y suis toujours resté) au départ en retraite d'André Mauzin, le 31 décembre 1966.

J'ai donc été l'un de ses proches collaborateurs durant un peu plus de 23 ans et je vais essayer de donner ici une idée de ce qu'on faisait à la SER dans l'une de ses deux missions essentielles : la stabilité du matériel roulant en ligne. L'autre mission était l'utilisation et l'entretien des deux, puis quatre voitures de mesure de l'état géométrique des voies qu'il avait inventées et réalisées, universellement nommées - et aujourd'hui encore - «voitures Mauzin ». Je n'y ai jamais participé et suis obligé de laisser à plus compétent cet aspect, mais, bien entendu, je connaissais parfaitement le principe de leur fonctionnement et je fus, autant qu'un chef de District, un lecteur assidu des kilomètres de graphiques qu'elles produisaient (le " Mauzin »!). En effet, avant tout essai de matériel en ligne, il fallait étudier ceux-ci de façon approfondie pour se faire une idée exacte de la qualité de la voie d'essai.

Il importe de préciser qu'à mon entrée à la SER l'essentiel des moyens de mesure existait depuis avant 1939 et que je les ai utilisés durant près de vingt ans sans grand changement. Ce n'est qu'à partir des années 1960 que des perfectionnements importants de ces moyens apparurent avec les jauges de contrainte.

Nous devons donc, avant toute évocation de la vie de la SER, entrer dans le détail de ces moyens de mesure qui sont vraiment l'œuvre essentielle d'A. Mauzin car, avant lui, il n'existait que des procédés rudimentaires et c'est avec lui que les mesures ferroviaires entrèrent dans le domaine scientifique et mesurable.

Dès avant 1939, ces moyens comportaient :

- deux voitures d'enregistrement " tout électrique » permettant 12 et 18 mesures simultanées sur papier photo,

- un meuble portatif à quatre enregistrements,

- des capteurs de force à quartz piézo-électrique,

- des accéléromètres à quartz piézo-électrique.

1- Les essais en ligne reprirent au printemps 1946. 
Jusqu'à la « fin du règne », de nombreux autres engins de mesure s'y ajoutèrent : deux voitures à seize enregistrements, un meuble à 16 voies et un à 8 voies.

Laissant à part les «meubles », d'appareillage identique à celui des voitures, il convient de s'étendre un peu sur celles-ci, dont les deux premières (celles d'avant-guerre) sont dues à un électricien de valeur : Renard, les deux dernières l'étant à un autre d'égale valeur, mais plus tard arrivé : Tarridec.

Une voiture était autonome sur sa batterie $24^{\mathrm{v}}$ au plomb ${ }^{2}$ qui alimentait une commutatrice $24^{\mathrm{v}}$ continu $/ 110^{\mathrm{v}}-50 \mathrm{~Hz}$. Les basses tensions (par exemple le chauffage des triodes, l'alimentation des « tubes électromètres » et des potentiomètres de mesure de déplacements) étaient prises sur la batterie. Les hautes tensions ( $1200^{\mathrm{v}}$ pour les oscillographes cathodiques) l'étaient sur le $110^{\mathrm{v}}$ avec stabilisation en intensité par tubes « fer-hydrogène » et en tension par « tubes au Néon ».

L'élément enregistreur était constitué de tubes cathodiques de diamètre $70 \mathrm{~mm}$ et, pour la répétition visuelle, de diamètre $120 \mathrm{~mm}$. A. Mauzin avait choisi ce "visualiseur» en raison de son extrême robustesse et de son aptitude à suivre des fréquences élevées.

Bien sûr, pour les usages courants, les fréquences examinées dépassaient rarement $10 \mathrm{~Hz}$ (vibrations verticales des caisses), mais le "meuble à 4 » avait la possibilité de dérouler son papier photo à $1 \mathrm{~m} / \mathrm{sec}$, d'où celle de lire jusqu'à $1000 / 1500 \mathrm{~Hz}$ (vibrations verticales des rails) $)^{3}$.

Le capteur essentiel, celui qui, pour la première fois, permit la mesure précise des efforts transversaux exercés par les essieux sur la voie ou, plus exactement, transmis aux essieux par les châssis (caisse ou bogie) des véhicules, est le capteur à quartz piézo-électrique.

Ces deux efforts diffèrent entre eux par l'effort d'inertie d'essieu qui a, à vrai dire, toujours échappé à ce genre de mesure. Les procédés actuels basés sur la déformation des toiles des roues en contiennent une partie mais je ne puis jurer qu'il soit pris en compte à $100 \%$ !

Pour l'utilisation pratique du quartz et de son effet piézo-électrique, A. Mauzin consulta une sommité des années 1930 : le professeur Langevin, mais en assura la mise au point complète. Le quartz comporte trois

2- Choisie pour la lenteur de sa courbe de décharge, malgré l'inconvénient de sa relative fragilité

3- Bien entendu, le dépouillement automatique et les spectres n'existaient pas à cette époque. Tout se faisait par lecture sur le papier. En ai-je «avalé», des kilomètres de bandes photo... 
inconvénients : grande dimension des disques de quartz ${ }^{4}(\varnothing=60 \mathrm{~mm}$ pour les locomotives) donc du capteur, obligation d'un électromètre car il s'agit ici d'électricité statique et, en corollaire, isolement parfait et blindage excellent de la partie électrostatique du circuit.

L'électromètre fut la tétrode du R. P. Lejay qui se révéla apte à bien résister aux secousses, chocs et vibrations, moyennant une suspension modeste.

En fait, ce système fonctionnait très bien et fut longtemps utilisé.

Les accéléromètres à inertie, ramenant la mesure de ã à celle de l'effort exercé par une masse sur des capteurs à quartz, furent d'abord énormes et forts lourds à manipuler car, ainsi que dans les capteurs d'efforts des essieux, on utilisait des piles de disques de quartz de faible sensibilité. Vers 1950, un très remarquable collaborateur d'A. Mauzin, André Chartet, dont nous reparlerons, eut l'idée d'employer des lames minces de quartz dites «en taille de Curie ", de sensibilité nettement supérieure, ce qui conduisit à des engins beaucoup plus pratiques et maniables.

Bien entendu, entre les tensions électriques brutes fournies par les divers capteurs et les plaques déflectrices des oscillographes se trouvaient intercalés des amplificateurs à un ou deux étages selon la sensibilité désirée. Lesquels ? Ma foi, à cette époque lointaine (nous allons d'avant 1939 à 1960 environ), c'étaient de bons vieux amplis à triodes 6 F 5 (provenance États-Unis), ces braves triodes dont on comprenait si bien le fonctionnement! Car je l'avoue à ma honte, je n'ai jamais pu assimiler comment marche un transistor... Et voici ma consolation :j’ai interrogé à leur sujet deux personnes de ma génération, un $\mathrm{X}+$ Télécom et un Sup Elec. Tous deux m'ont fait le même aveu. Pour prouver que ça ne doit pas être si évident que cela, je citerai les études récentes d'ingénieur de la Marine nationale faites vers 1983 par mon fils à l'École militaire de la flotte. On leur a tout bonnement présenté cet engin comme un «tripôle » obéissant à certaines équations. Au moins, c’est clair et limpide !

Pour les efforts transversaux d'essieux, l'un des buts fondamentaux de la SER, on les mesura systématiquement sur les locomotives de série anciennes (2D2) en vue de l'amélioration de leur tenue et, surtout, sur tous les engins moteurs prototypes dont la liste complète serait fort longue. Citons seulement les 241C1 (vapeur), CC 6001, BBB 6002, BB 8100, CC 7100, BB 9004, BB 16500 qui nous occupèrent durant de longues années, sans parler des voitures et wagons.

4- Pour une simple question de résistance mécanique. 
Chaque ancienne ou nouvelle série nécessitait une étude particulière d'adaptation de gros capteurs à quartz de $60 \mathrm{~mm}$ qui constituait pour notre bureau d'études un «pain quotidien » perpétuellement renouvelé, donc des études en général fort intéressantes qui soutenaient l'émulation de nos dessinateurs, souvent remarquables, sous la direction de chefs d'études eux-mêmes remarquables (Duron, Lachaud, Conrairie).

Dans le cas d'essieux à boîtes intérieures (essieux moteurs et de bogie des 2D2 ou de locomotives à vapeur), les montages étaient souvent fort compliqués, assez délicats d'utilisation et d'une grande variété.

Dans le cas de locomotives électriques plus récentes, à bogies tous moteurs et à boîtes extérieures, ils étaient nettement plus simples et nous citerons celui du prototype (sans suite) BBB 6002 qui fonctionna très bien et avec lequel je fis en 1950, avec quelle fierté ! mes premières armes d'essayeur de locomotives, après avoir fait mon apprentissage comme essayeur de voitures du seul point de vue de leur confort, chose évidemment bien plus facile (fig. 1).

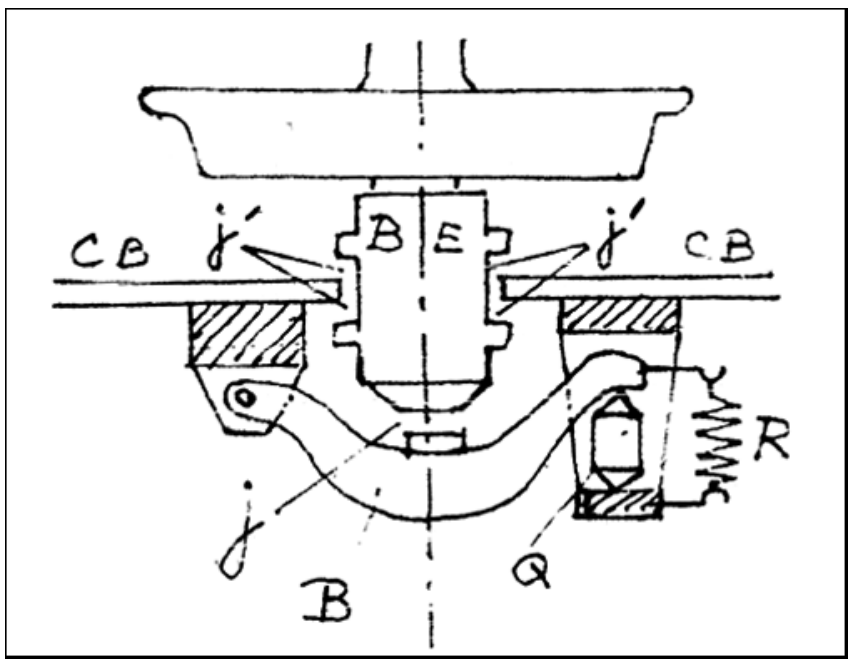

Figure 1.

On agrandit les jeux transversaux entre boîte d'essieu BE et châssis de bogie $\mathrm{CB}$ de façon à obtenir $: j^{\prime}=j+10 \mathrm{~mm}, j$ étant le jeu normal, ceci pour éviter toute dérivation de l'effort transversal dans son passage de la boîte à ce châssis, le jeu correct j étant ménagé entre celle-ci et le balancier B. Le capteur à quartz est en Q. Un ressort de rappel R (de faible raideur) assure le contact permanent sur ce capteur. 
C'est fort simple mais, comme dit plus haut, il a existé des montages beaucoup plus compliqués et d'emploi parfois délicat.

Tout cela étant dit, quelle était la doctrine d'A. Mauzin en matière de suspension entre caisse et bogie?

\section{Suspension transversale}

Je ne pense pas qu'il se soit livré à de très savants calculs concernant le lacet, problème fort difficile et dont la solution, approchée mais correcte, ne fut trouvée que durant les dix dernières années de son règne (à l'exception d'une étude de jeunesse dont je parlerai plus loin), mais je ne puis être très affirmatif à ce point de vue, car il fut, durant ces années-là, président du Comité ORE - B52 dont les travaux concernaient précisément ce problème théorique. M'occupant beaucoup des essais en ligne, je n'ai suivi ces travaux que de loin.

Toujours est-il que, tant pour le matériel moteur que pour le remorqué, je lui ai bien souvent entendu préconiser une suspension transversale pendulaire à pendules d'au moins $50 \mathrm{~cm}$ de longueur, ou l'équivalent sous forme de ressorts de rappel, ou blocs de caoutchouc en sandwich (dans les dernières années), avec jeux transversaux importants. Cette liaison conférait aux trois fréquences propres de déplacement transversal de caisse - deux dites de «ballant-roulis » couplées entre elles et une de lacet purement angulaire - des valeurs suffisamment basses pour que l'excitation provenant des bogies passe les trois résonances à vitesse relativement faible. C'est ce qu'on appelait les « conditions supercritiques ».

Il n'était d'ailleurs pas le seul à raisonner ainsi et, sur ce point, il était en total accord avec les Allemands, alors représentés par le Dr Müller, avec qui d'ailleurs il s'entendait le mieux du monde et qui était un homme charmant que j'ai bien connu.

A cette époque et pour les voitures, les bielles de liaison transversales étaient, sauf rarissimes exceptions, bien plus courtes que $50 \mathrm{~cm}$.

\section{Suspension verticale}

Il s'agit ici des voitures car les locomotives ont forcément une suspension verticale assez raide. Là, il préconisait une fréquence propre de l'ordre de $1 \mathrm{~Hz}$, alors que toutes les voitures d'alors avaient une fréquence de l'ordre de $2 \mathrm{~Hz}$, entrant fâcheusement en résonance avec le passage des joints de rails aux vitesses de :

- $119 \mathrm{~km} / \mathrm{h}$ sur rails de 16,5 m (cas du Sud-Ouest)

- $130 \mathrm{~km} / \mathrm{h}$ sur rails de $18 \mathrm{~m}$ (qu'on commençait à poser). 
Là aussi, il n'était pas le seul à penser ainsi car, vers 1950, les CFF (Chemins de fer fédéraux suisses) lancèrent des suspensions très souples, avec amortisseurs hydrauliques, qui battaient de loin les françaises (essais SNCF avec Guignard, chef des voitures et wagons des CFF).

J'ai réservé plus haut le cas d'une étude sur le lacet de (quasi) jeunesse d'A. Mauzin (cet article n'a pas été publié dans la Revue générale des chemins de fer. Date probable : 1935-1936) et, ici, vous me permettrez d'égayer un peu ce « topo » assez aride par une histoire amusante concernant cette étude. Ce faisant je me moquerai un peu de mon vieux Maitre, oh! pas méchamment ! qu'on veuille me le pardonner. Ce fut d'ailleurs, à ma connaissance, le seul cas où il se trompa lourdement.

À qui ce n'est-il arrivé ? À commencer par moi... qui eus quelques occasions de battre ma coulpe!

Donc, il chercha, sans recourir à la Dynamique, à calculer la longueur d'onde du lacet d'un bogie à deux essieux liés rigidement au châssis.

On ne peut lui reprocher d'avoir admis une loi de force au contact roue/rail complètement fausse car, à cette époque, personne ne connaissait quoi que ce soit sur cette question. Ce n'est, je pense, que vers 1939-1940 que le Hollandais Labrijn commença ses expériences sur le roulement oblique d'un essieu qui, vers 1942, aboutirent au réseau des célèbres courbes « de pseudo-glissement » rendues définitives après les mesures de Müller à la DB (Deutsche Bahn). A. Mauzin admit donc une loi de frottement sec de glissement roue/rail constant et indépendant de la direction du roulement ${ }^{5}$ et, ensuite, il innova en posant le principe suivant : « le système suit une trajectoire telle que le travail des forces de frottement est minimum.»

Ceci semble le bon sens et l'évidence mêmes. Le seul petit ennui, c'est que ce principe n'existe pas en mécanique et, donc, est archi-faux ! Qu'on se donne la peine de consulter les cours magistraux de mécanique rationnelle, ceux de l'X par exemple...

Suivaient deux pages d'algèbre qui, au moins, paraissaient inattaquables.

Parmi les nombreux lecteurs ${ }^{6}$ de cette très sérieuse étude, un seul eut le courage de refaire tous les calculs : moi !

Et c'est là que réside le côté cocasse de cette histoire !

5- Sauf quand l'angle d'attaque est nul.

6- Peut-être assez distraits... 
Il faut dire ici, ce que je savais par longue expérience, que ce brave A. Mauzin... écrivait comme un chat, surtout lorsqu'il brouillonnait ! Il confondit plusieurs fois « $\mathrm{e}$ » (écartement de la voie) et « $1 »(1 / 2 \mathrm{em}-$ pattement du bogie), selon qu'il les écrivait plus ou moins allongés au gré de sa fantaisie. Il en résulta un «horrible mélange », tel que celui d'« os et de chairs meurtris » de la pauvre Mme Jézabel!

Il obtint une formule... totalement fausse bien sûr mais qui satisfaisait à ce qui constituait son souci majeur lorsqu'il calculait : l'homogénéité. Il est évident que confondre une longueur avec une autre ne l'altère en aucune façon!

Alors, intervint la chance proverbiale qui lui sourit sans faille durant toute sa carrière, car, pour un bogie courant de cette époque, « 1 » était compris entre $1,25 \mathrm{~m}$ et $1,5 \mathrm{~m}$, mettons $1,35 \mathrm{~m}$ en moyenne. Or, «e », écartement de la voie, est de 1, $43 \mathrm{~m}$ et la différence est bien faible !

Et puis, après tout, son principe de travail minimum des frottements n'est peut-être pas tellement loin de la vérité vraie..., au moins pour un système.

Conclusion : sa formule donnait des résultats fort vraisemblables !

J'en fis, sur sa demande, une application merveilleuse au cas d'une voiture à deux essieux à suspension transversale par sandwiches de caoutchouc dont Kléber-Colombes nous proposa l'essai et qui eut, hélas, un lacet phénoménal! je me souviens encore de ma frayeur lorsque, à $\mathrm{V}=140$ sur le viaduc de Barentin (Rouen-Le Havre), la balustrade dudit viaduc se rapprochait périodiquement de nous, à nous faire craindre que nous allions nous envoler par-dessus ! c'est que Kléber comptait sur l'amortissement interne du caoutchouc... et que celui-ci faisait, au contraire, cruellement défaut.

Mon vieux Maître (il était près de son départ) me pria d'appliquer la fameuse formule, à laquelle il faisait encore toute confiance, au cas de cette voiture à deux essieux considérée comme un grand bogie, donc l >> e, et le calcul donna un résultat parfaitement exact par rapport à celui de l'essai en ce qui concerne la longueur d'onde du lacet.

Il y a des gens « nés coiffés »!

A l'époque de ma découverte, ce brave homme vivait encore et à quoi bon ébruiter cela ?

Maintenant, le cas n'est plus le même... et puis cette anecdote est si croustillante que je ne puis résister au plaisir (sans aucun fiel) de la conter. 
Passons maintenant à l'organisation matérielle de la SER. A. Mauzin a toujours voulu qu'elle forme un organisme entièrement autonome pouvant se suffire à lui-même, avec bureau d'études et atelier, afin qu'en cas d'urgence on n'ait pas à recourir, avec les délais inévitables, à des études et confections à l'extérieur.

Le tout tenait, au 55 boulevard de la Gare (pas encore "Auriolisé ») à Paris XIII, dans un vieux petit bâtiment d'un rez-dechaussée et d'un étage ${ }^{7}$ qui, du temps des chevaux, servait de réserve à fourrage pour les besoins de la gare des marchandises.

Il comprenait :

- un atelier bien équipé en machines-outils,

- un bureau d'études bien étoffé (déjà cité),

- un laboratoire d'électricité-électronique où s'élaboraient les câblages des voitures et meubles d'enregistrement (Tarridec),

- un laboratoire des capteurs (Servant, puis Jagut),

- des chefs d'essais : Roucayrols et moi pour les essais en ligne, Servant pour ceux «à poste fixe » tels que ceux de flambement de voie effectués en 1947 sur demande de Robert Lévi. Après leur départ, ceux-ci (restés pour moi de vieux amis) furent remplacés par Laurent, Malige, Vattéoni et Biau. Tous furent remarquables.

Je tiens aussi à rendre hommage au personnel d'exécution de la SER, en particulier à celui des essais en ligne, qui m'a toujours assisté avec compétence, dévouement et bonne humeur. Il était, Dieu merci, beaucoup moins revendicatif qu'en l'an de grâce 2000 (à une certaine époque, après la guerre, nous avons travaillé $9 \times 6=54$ heures par semaine). Les deux seuls survivants de ma plus vieille équipe sont restés pour moi des amis.

- Un service voie (Baehr), que je cite pour mémoire,

- des études spéciales : elles étaient confiées à un seul, mais quel personnage hors du commun : André Chartet $(\mathrm{X})$ ! De première force en maths et mécanique rationnelle, il était de plus - ce qui ne gâte jamais rien - un parfait galant homme! Durant son séjour assez bref à la SER, il s'illustra par :

. une communication à l'Académie des sciences sur le roulement oblique de deux cylindres,

. une étude extrêmement savante sur les conditions de déraillement d'un essieu (voir RGCF d'août 1951), et une autre sur les suspensions verticales (RGCF de novembre 1952),

7- Sous toiture, il y faisait jusqu’à $33^{\circ}$ en été, bien entendu sans la moindre réfrigération... 
l'étude non moins savante d'une « voiture pendulaire » aux bogies des plus originaux (mais hélas trop fragiles) en collaboration avec notre patron.

Il dirigea ensuite le banc d'essais de Vitry-sur-Seine, puis quitta la SNCF, en quoi il eut grand tort, car la plus brillante des carrières lui était probablement assurée s'il était resté. Bien après lui, un habile théoricien, Joly, établit les sept équations différentielles qui régissent, avec une bonne approximation, le mouvement de lacet d'un bogie en tenant compte des élasticités des liaisons longitudinales et transversales entre boites d'essieu et châssis.

Puis, grâce au don par le cher M. Nouvion (toujours si gentil avec nous qui lui essayions en ligne ses nouvelles locomotives) d'une calculatrice analogique, Joly créa l'embryon d'un centre de calcul qui fut ensuite beaucoup développé, mais ceci après le départ d’A. Mauzin.

Le bureau administratif était aussi réduit que possible... et sans chef de bureau (pas d'éminence grise!). Preuve de grand bon sens!

Il est probable que cette SER, vivant indépendante et en autarcie, souleva quelques jalousies de la part de ceux qui, suivant les vues du grand Armand du Plessis ${ }^{8}$, ne voulaient pas « d'États dans l'État »...

J'en peux fournir deux preuves qui me paraissent assez claires.

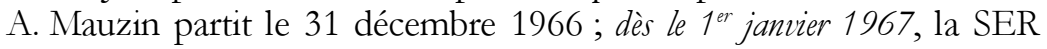
était rattachée à la division des Essais de matériel (chef Maurice Laplaiche). Le même Laplaiche me dit, en 1968: «il faut que nous vous digérions et vous serez digérés !», ceci avec un air gourmand !... C'était charmant ! Et pourtant, je m'entendis bien avec lui (bien qu'il fût parfois assez fort en $\mathrm{g} . .$.$) car, au fond, c'était un très brave homme et je$ restai en relations amicales avec lui jusqu'à sa mort.

Mais, enfin, la SER, "l'incorrigible indépendante » avait bel et bien cessé d'exister avec son vieux patron! Elle ne ressuscita jamais.

Ce qu'on ne peut lui contester, en toute vérité, c'est qu'elle a existé et œuvré pour le bien du chemin de fer de 1927 à 1966. 39 ans !

André Mauzin ayant consacré sa vie à la fois à la voie et à la traction, il était naturel qu'il essayât de rapprocher ces deux services qui ont tant de problèmes à débattre en commun. De là, en collaboration avec le chef des Installations fixes Feyrabend, la création du Comité de liaison Traction-Voie qui se réunissait tous les mois. Les quelques séances auxquelles j’assistai furent toujours du plus haut intérêt. Prions Dieu que cette institution existe encore !... Amen.

8- Alias cardinal duc de Richelieu. 
Il me reste à parler des qualités intellectuelles et humaines d'A. Mauzin.

Il est certain qu'il avait, pour toutes les questions de mécanique, une incroyable intuition venant plutôt de son génie propre que de savants calculs. C'était de plus un fort brave homme (parfois un peu nerveux, c'est vrai, mais nul n'est parfait ici-bas !) et les rapports avec lui étaient des plus directs et aisés pour tous ses collaborateurs.

A mon avis, sa plus grande qualité était son art d'éveiller et d'entretenir l'enthousiasme autour de lui.

Je restai son ami fidèle jusqu'à sa mort, à l'âge de 93 ans, et je lui dois beaucoup de reconnaissance que je suis heureux de manifester publiquement ici. 